\title{
The ethics of unsolicited diagnosis of mental disorder in acquaintances: benefits and dangers ${ }^{\dagger}$
}

\author{
Edward W. Mitchell
}

The Psychiatrist (2011), 35, 297-301, doi: 10.1192/pb.bp.110.032953

${ }^{1}$ Warneford Hospital, Oxford, UK Correspondence to Edward W. Mitchell (mitchell@fulbrightmail.org) First received 29 Sep 2010, final revision 27 Nov, accepted 13 Jan 2011

\begin{abstract}
Summary I examine here the activity of 'unsolicited diagnosis' of psychiatric disorder - the act of informing a person that they show signs and symptoms of mental disorder, outside of a patient-professional relationship. Whether unsolicited psychiatric diagnosis is a legitimate ethical activity for psychiatrists (and other healthcare professionals) in which to engage is an issue founded upon the trade-off between potential benefits and harm to the recipient of the diagnosis. However, potential harm specific to a psychiatric diagnosis (such as issues related to stigma, confidentiality and paternalism) suggests that making unsolicited diagnoses of psychiatric disorder is even more ethically fraught than making unsolicited diagnoses of physical disorder.
\end{abstract}

\section{Declaration of interest None.}

A doctor is duty bound to assist in an emergency situation, yet there is a paucity of literature on the moral and professional obligations of doctors when they observe signs and symptoms of disorder in those who have not approached them for assistance in non-emergency situations. I have examined elsewhere whether doctors should make unsolicited or 'passer-by' diagnoses of illness in persons encountered outside of a professional environment, who have not sought help from that doctor. ${ }^{1}$ This article generated considerable feedback from doctors who had made spot diagnoses and who in some cases felt that their action might have saved lives. The lay press also details the gratitude of people diagnosed with serious disease by good Samaritan doctors, who detected clinical signs when shaking their hand, seeing them on television, or even chancing upon their portrait at an art gallery. ${ }^{2-4}$ Such situations differ from 'off-the-cuff consultations', ${ }^{5}$ in which the person requests help or advice, stereotypically by interjection during dinner-party small talk.

It was striking that among these many stories of cancers, hypothyroidism, acromegaly and suspicious moles, I have not heard one in which a doctor made an unsolicited diagnosis of psychiatric disorder. And yet one does not need to talk to many doctors before finding one who wonders whether he or she should have said something to a colleague who showed significant signs of behavioural disturbance before they died by suicide. Furthermore, in casual conversations with doctors, psychiatric diagnoses are meted out to a wide range of persons (not always flippantly), including colleagues, friends, family, acquaintances and

'See editorial, pp. 281-282, this issue. partners. If up to $50 \%$ of people develop a mental illness during their lifetime, ${ }^{6}$ such statements will not uncommonly be correct. Yet they are rarely, if ever, mentioned to the person about whom the comment is made.

Some case examples help us to imagine situations in which informal psychiatric diagnoses might be made.

\section{Case vignette 1}

A colleague, normally cheerful and outgoing, over the past 6 months has become gradually withdrawn and solemn. He has lost weight, and has occasionally appeared tearful at work. When you enquire as to how he is feeling, he tells you of waking early and excessive sleepiness in the day. Do you implore him to go to see his general practitioner, believing him to be suffering from depression?

\section{Case vignette 2}

\begin{abstract}
Your son's housemate at university has begun acting bizarrely. Once an active member of the drama group and an academically successful individual, he now barely leaves his room, which is dirty and piled high with unreturned library books. Collecting your son from university, you noticed his friend was very distressed and frequently referred to particular websites and books which he says contain coded messages for terrorists. Do you advise your son to try to get his friend to see a doctor in case he has a psychotic illness?
\end{abstract}

\section{Case vignette 3}

A friend mentions he is having relationship problems. His girlfriend is controlling and jealous of him, and prone to sudden temper outbursts and continuous arguments. She has threatened suicide to prevent him leaving her. You wonder 
whether she may have borderline personality disorder and benefit from, for example, a complex needs psychotherapeutic approach; do you tell your friend?

\section{Potential benefits of diagnosis}

Stories from doctors and the press, which detail serious disease potentially cured or ameliorated as a result of intervention by hawk-eyed doctors, reveal the most important function provided by an unsolicited diagnosis: it is a formulation which opens up a route to formal diagnosis and treatment through contact with health professionals. In the case of a colleague showing behavioural disturbance, the doctor standing by and doing nothing out of concern for offending his colleague or for his own sense of professionalism may be making the greater error. Concern for psychiatric colleagues might take into account the fact that psychiatrists have one of the highest suicide rates among medical specialties. ${ }^{7}$ Encouragement to seek help does not require a diagnosis; a humane concern for the suffering of others and a sympathetic ear is more appropriate, even if that offer turns out to be unappreciated. Indeed, should we suspect a colleague's behaviour or judgement to be sufficiently disturbed that it may impair their fitness to practise, licensing bodies such as the General Medical Council require us to address the issue with the person or appropriate manager.

Facilitation of such contact is of particular importance in the case of mental health problems. Even though the lifetime prevalence of mental disorder is estimated to be $50 \%$ of individuals, only a small proportion receive professional treatment in any year, even among those reporting substantial distress and role impairment. ${ }^{8}$ Indeed, only about a half of individuals with addictive disorders or phobias ever receive treatment during their lifetime, and there is a median delay from symptom development to treatment of 6 years for major depression and 14 years for generalised anxiety disorder. ${ }^{9}$ This is especially worrying considering that for some disorders such as schizophrenia delay in treatment is associated with a poorer outcome. ${ }^{10}$ People are frequently reluctant to disclose psychological symptoms in general practice consultations, ${ }^{11}$ either on account of stigma or believing that such symptoms are 'part of life' rather than representing a potential health problem. Furthermore, the public deems self-help to be the most efficacious intervention for psychiatric disorder; ${ }^{12}$ even when people do enlist the help of others, they do not necessarily see health professionals as their first port of call. In the USA, a quarter of those who seek help turn to clergy, whereas psychiatrists and medical doctors are contacted by only $17 \% .^{13}$ The public may regard psychiatry with suspicion and its methods as harmful ${ }^{12}$ and, as a consequence, they are reluctant to seek help or adhere to treatment. ${ }^{14}$ However, they are more likely to seek help when told to do so by another person. ${ }^{15}$

Psychiatric conditions have a particularly poor prognosis, increasing the potential benefits of (and thereby lowering the threshold for) unsolicited diagnosis. For example, approximately 1 in every 20 people with depression, alcoholism or schizophrenia kills themselves (with the greatest risk being soon after symptom onset). ${ }^{16}$
The possibility of risk of harm to others (albeit highly unlikely in most cases of mental disorder) presents a particular diagnostic exigency which is not shared with physical conditions (save for certain infectious diseases and conditions impairing ability to drive). ${ }^{17}$ Mental disorder also carries a high social burden to friends, family and others.

In summary, the benefits to the recipient of an unsolicited diagnosis may include a substantial decrease in both morbidity and mortality. There may also be benefits to society through decreased social burden. Other significant primary and secondary gains may emerge, most notably the sense of relief and removal of responsibility for 'bad' behaviour which are a function of gaining a medical 'label' for what might otherwise be seen by the self and others as 'deviant' behaviour. ${ }^{18}$

\section{Risk of harm}

Unsolicited diagnosis also carries a risk of harm. Type I errors (false-positive diagnoses, which hold the potential to cause their recipient grave psychological and physical harm) are a risk of any diagnosis, no matter whether of physical or mental disorder, and whether solicited or not. However, psychiatric diagnosis, due to its reliance upon a process of extensive clinical history and mental state examination (which is likely impractical or inappropriate in an informal setting) may be particularly liable to type I error; even in formal settings, the majority of positives generated by brief psychiatric screening instruments are false positives (although, in fact, the positive predictive value of such instruments is no worse than for physical screening tests such as faecal occult blood testing or mammography). ${ }^{19}$ Two types of false positive errors may be made by the diagnostician: (1) wrongly attributing psychiatric disorder to a person; and (2) correctly attributing psychiatric disorder, but being mistaken as to the actual diagnosis.

It could be countered that owing to their necessary foundation on less than optimal clinical information, both psychiatric and physical diagnoses will be accordingly inaccurate to greater or lesser degrees when made in informal contexts. For example, a formal diagnosis of myocardial infarction, in addition to clinical history, requires abnormal electrocardiographic activity and/or elevated serum cardiac markers, ${ }^{20}$ which are clearly not data that can be gleaned in an informal setting. That does not, however, mean that making an unsolicited diagnosis of a myocardial infarction upon seeing a breathless man gripping his chest is clinically presumptive, if the aim of making it is to set in train a series of events that will result in the person receiving formal diagnosis and treatment. Likewise, it does not matter when an unsolicited diagnosis of major depression in fact turns out to be bipolar disorder or schizoaffective disorder, if it spurs its recipient into getting treatment. Thus, although psychiatric diagnoses (whether solicited or not) are frequently criticised for having poor interrater reliability (and, indeed, validity), ${ }^{21}$ this does not necessarily undermine their utility ${ }^{22}$ if they eventually result in benefit for the recipient.

Mistaking a condition for one more serious than that by which a person is actually affected can cause particular problems for the person for whom the diagnosis is made. 
A goitre may indicate thyroid cancer but is more likely caused by thyroiditis; flattened affect could indicate schizophrenia or mild depression. If the goal of unsolicited diagnosis is as catalyst to formal diagnosis and treatment, then the graver diagnosis comes with a correspondingly increased risk of maleficence. This suggests that circumlocutory language of a formulation would be more appropriate than a specific diagnosis in most cases, pointing out the possibility of a problem potentially amenable to treatment and encouraging contact with healthcare professionals in general terms (inaccurate diagnoses may also be subsequently presented as a 'fait accompli' by their recipient, prejudicing further diagnosis and treatment).

Some such risks can be offset if the diagnostician has a level of intimacy with the person for whom they make a diagnosis. Indeed, in the case of unsolicited diagnoses of both psychiatric and physical disorder, applying one's expertise to a person with whom we are familiar is likely to be seen by doctors as more ethically palatable than doing so for a relative stranger. In the case of unsolicited psychiatric diagnosis, only in the context of such intimacy is the diagnostician likely able to obtain a reasonable history and mental state examination. Low mood and trouble sleeping would be unreliable signs of depression if confided by a casual acquaintance without context; the same symptoms will carry far greater probative value if observed over a period of time, and apparently without cause, in person whom the diagnostician knows intimately (although psychiatric examination of such persons carries its own ethical issues).

Other situations may be envisaged where harm is likely to result from unsolicited diagnosis, for example, when making an unsolicited diagnosis of a condition which the person has already presented to a health professional. It is easy to see the potential stress caused by telling a person that they show signs of mental disorder, when they had already sought help for such problems. Indeed, there might be a general presumption that behaviour abnormal enough to draw an unsolicited diagnosis might already have resulted in presentation to a health professional, on account of the public's ability to recognise mental disorder (even if they struggle to differentiate between various disorders). ${ }^{12}$ If accepted, this hypothesis would considerably reduce the need for, and ethical acceptability of, unsolicited diagnosis.

An unsolicited diagnosis will not be of benefit to the person who is not likely, or not able, to follow it up, either through incapacity or inability to access suitable healthcare resources. Those who have egosyntonic psychiatric symptoms (or who derive significant primary or secondary gains from their symptoms) might be particularly unlikely to follow up an unsolicited diagnosis. For example, van Putten and colleagues $^{23}$ identified a group of psychotic patients who did not adhere to pharmacotherapy because they preferred their grandiose psychotic state over a medicated but sober reality. The inability of such persons to benefit from downstream 'formal' diagnoses and treatment leaves potential for harm as the only consequence of unsolicited diagnosis.

There is also great potential for 'downside' and little 'upside' to making unsolicited diagnoses, if the condition being diagnosed has a particularly poor prognosis, or little exists by way of treatment or palliation.

\section{Types of harm particular to unsolicited psychiatric diagnosis}

Aside from the usual negative effects of receiving a diagnosis of illness (a potentially significant source of stress and worry), differences in the social consequences of being diagnosed with psychiatric and physical disorder entail different ethical considerations for the diagnostician in formal and informal settings.

The first issue to be considered is stigma. People with mental disorder may face negative attitudes and discrimination from other members of society. ${ }^{24}$ That the diagnostician may not him- or herself attach stigma to the person's diagnosis is of no consequence (although it is a worrying finding that health professionals may themselves perpetuate stigma towards people with mental disorder). ${ }^{25}$ If the person holds negative beliefs about individuals with mental disorder, and as a result of unsolicited diagnosis believes that they might be one of those people, they are liable to hold (or believe that others hold) negative beliefs towards themselves. This 'self-stigma' results in loss of selfesteem and self-efficacy, and is associated with poorer clinical outcomes. ${ }^{26,27}$

Concern about confidentiality is another source of specific potential harm. Mental health (along with sexual/ reproductive health and drug use) is a category of health information with which people have particular confidentiality concerns. ${ }^{28}$ By having an unsolicited diagnosis made in an informal setting, the person might presume that the diagnostician could be equally as informal in respect of confidentiality with regard to that diagnosis, especially as some patients believe (sometimes correctly) that doctors and nurses share confidential medical information with family and friends. ${ }^{29}$

Finally, it might be argued that an unsolicited psychiatric diagnosis presents the opportunity for the health professional to commit a particularly grave transgression of the limits of his or her profession that an unsolicited physical diagnosis does not.

\section{Limits of practice}

The transgression of the limits of practice is a function of differing views about treatment for physical and psychiatric conditions in the mind of the public and many professionals. Harm may of course occur to persons with solicited or unsolicited physical diagnoses (particularly through adverse effects of pharmaceutical or surgical intervention), but the public holds more positive attitudes towards treatment for physical conditions than for psychiatric conditions. $^{30}$ Popular images of psychiatric treatment focus on negative and paternalistic issues such as involuntary detention and electroconvulsive therapy. ${ }^{12}$

In the same way, members of society, researchers, and mental health professionals of a critical or anti-psychiatry persuasion, ${ }^{31}$ who concentrate on the adverse effects of diagnosis and treatment, might regard the labelling of the individual through unsolicited psychiatric diagnosis as 
particularly unpalatable and paternalistic; that the diagnosis carries a high risk of being incorrect or a false positive is likely to prove doubly so. The situation differs from that in which a person voluntarily seeks a diagnosis on account of distress from symptoms of mental disorder (although those critical of the consequences of psychiatric diagnosis would argue that both situations result in an unnecessary medicalisation of 'problems of living'). ${ }^{32}$

Such notions echo the downstream consequences of receiving a 'dangerous and severe personality disorder' label in the UK, an unsought 'diagnosis' which can result in potential harm through deprivation of liberty, and one which has resulted in considerable introspection by the psychiatric profession as to their limits of practice. ${ }^{33}$

As diagnoses can harm their recipients, so can they be of harm to those who award them. Delivering unsolicited diagnoses to strangers fits with a popular conception of psychiatrists constantly analysing everyone they encounter, and is thus hardly compatible with attempts by psychiatrists to portray themselves as professionals whose special training does not cause them to somehow judge or monitor the behaviour and utterances of others. Indeed, the American Psychiatric Association deems that 'it is unethical for a psychiatrist to offer a professional opinion unless he or she has conducted an examination and has been granted proper authorization'. ${ }^{34}$ This so-called 'Goldwater rule' was designed to apply to psychiatric analysis of public figures following publication of a damning survey of psychiatrists into the psychological characteristics of the US presidential candidate, Barry Goldwater. ${ }^{35}$ It does highlight, however, the profession's rightful concern with causing negative effects upon an individual through flippant commentary or without in-depth knowledge of an individual case.

\section{Conclusions}

Unsolicited psychiatric diagnosis is the activity of diagnosing mental disorder in persons with whom the diagnostician is acquainted outside of a patientprofessional relationship, but who have not solicited the diagnostician's help. As regards its ethicality, it is an activity that shares much with the unsolicited or 'passer-by' diagnosis of physical conditions; however, unsolicited diagnosis of psychiatric conditions raises particular issues pertaining to confidentiality, stigma, accuracy of diagnosis, and whether the resultant labelling of individuals who had not sought help for their problems transgresses the limits of psychiatric practice. Instances of unsolicited diagnosis of both physical and mental disorders would seem to be sanctioned by a pragmatic 'ethics of care' approach rather than a deontological code of ethics. However, the thorny ethical issues involved in unsolicited psychiatric diagnosis in particular would seem to caution us to view it as an activity whose practise should be left to the particularly brave (or foolhardy) health professional, or one finding him- or herself in a situation of particular exigency, such as in the case of an obviously disturbed colleague.

\section{Acknowledgements}

The author acknowledges the help of Professor Tony Hope and Professor Mike Parker at the Ethox Centre, University of Oxford, as well as Professor
Tom Gutheil, Professor Harold Bursztajn and the Program in Psychiatry Law at Harvard Medical School, USA, for their comments on drafts of this manuscript. This paper was written while on elective funded by an Institute of Medical Ethics elective bursary.

\section{About the author}

Edward W. Mitchell MA MPhil PhD BMBCh is a core trainee (CT1) in psychiatry at the Warneford Hospital, Oxford and Buckinghamshire Mental Health NHS Foundation Trust, Oxford, UK.

\section{References}

1 Mitchell EW. The ethics of passer-by diagnosis. Lancet 2008; 371: 85-7.

2 BBC News. Handshake diagnosed brain tumour. BBC News 2008; 15 February (http://news.bbc.co.uk/1/hi/health/7246419.stm)

3 BBC News. Irish minister in TV tumour spot. BBC News 2007; 31 January (http://news.bbc.co.uk/1/hi/world/europe/6318893.stm).

4 Hughes R. Close encounters. Time 1998; 13 April (http:// www.time.com/time/magazine/article/0,9171,988127,00.html).

5 Weingarten M. Off the cuff consultations. BMJ 1985; 291: 1321-2.

6 Kessler RC, McGonagle KA, Zhao S, Nelson CB, Hughes M, Eshleman S, et al. Lifetime and 12-month prevalence of DSM-III-R psychiatric disorders in the United States: results from the National Comorbidity Survey. Arch Gen Psychiatry 1994; 51: 8-19.

7 Hawton K, Clements A, Sakarovitch C, Simkin S, Deeks J. Suicide in doctors: a study of risk according to gender, seniority and specialty in medical practitioners in England and Wales, 1979-1995. J Epidemiol Community Health 2001; 55: 296-300.

8 Katz SJ, Kessler RC, Frank RG, Leaf P, Lin E, Edlund M. The use of outpatient mental health services in the United States and Ontario: the impact of mental morbidity and perceived need for care. Am J Public Health 1997; 87: 1136-43.

9 Kessler RC, Olfson M, Berglund PA. Patterns and predictors of treatment contact after first onset of psychiatric disorders. Am J Psychiatry 1998; 155: 62-9.

10 Harrigan S, McGorry P, Krstev H. Does treatment delay in first-episode psychosis really matter? Psychol Med 2003; 33: 97-110.

11 Prior L, Wood F, Lewis G, Pill R. Stigma revisited: disclosure of emotional problems in primary care consultations in Wales. Soc Sci Med 2003; 56: 2191-200.

12 Jorm AF. Mental health literacy. Public knowledge and beliefs about mental disorders. Br J Psychiatry 2000; 177: 396-401.

13 Wang PS, Berglund PA, Kessler RC. Patterns and correlates of contacting clergy for mental disorders in the United States. Health Serv Res 2003; 38: 647-73.

14 Fischer W, Goerg D, Zbinden E, Guimón J. Determining factors and the effects of attitudes towards psychotropic medication. In The Image of Madness: The Public Facing Mental Illness and Psychiatric Treatment (eds J Guimón, W Fischer, N Sartorius): 162-86. Karger, 1999.

15 Vogel DL, Wade NG, Wester SR, Larson L, Hackler AH. Seeking help from a mental health professional: the influence of one's social network. J Clin Psychol 2007; 63: 233-45.

16 Inskip HM, Harris EC, Barraclough B. Lifetime risk of suicide for affective disorder, alcoholism and schizophrenia. Br J Psychiatry 1998; 172: 35-7.

17 Mitchell EW. Does psychiatric disorder affect the likelihood of violent offending? A critique of the major findings. Med Sci Law 1999; 39: 2330.

18 Chadwick PK. Schizophrenia: The Positive Perspective. Routledge, 1997.

19 Leon AC, Portera L, Olfson M, Weissman MM, Kathol RG, Farber L, et al. False positive results: a challenge for psychiatric screening in primary care. Am J Psychiatry 1997; 154: 1462-4.

20 Gillum RF, Fortmann SP, Prineas RJ, Kottke TE. International diagnostic criteria for acute myocardial infarction and acute stroke. Am Heart J 1984; 108: 150-8. 
21 Spitzer RL, Fleiss JL. A re-analysis of the reliability of psychiatric diagnosis. Br J Psychiatry 1974; 125: 341-7.

22 Kendell R, Jablensky A. Distinguishing between the validity and utility of psychiatric diagnoses. Am J Psychiatry 2003; 160: 4-12.

23 van Putten T, Crumpton E, Yale C. Drug refusal in schizophrenics and the wish to be crazy. Arch Gen Psychiatry 1976; 33: 1443-6.

24 Crisp AH, Gelder MG, Rix S, Meltzer HI, Rowlands OJ. Stigmatisation of people with mental illnesses. Br J Psychiatry 2000; 177: 4-7.

25 Corker E. Stigma caused by psychiatrists (letter). Br J Psychiatry 2001; 178: 379

26 Watson AC, Corrigan P, Larson JE, Sells M. Self-stigma in people with mental illness. Schizophr Bull 2007; 33: 1312-8.

27 Corrigan PW, Watson AC. Understanding the impact of stigma on people with mental illness. World Psychiatry 2002; 1: 16-20.

28 Sankar P, Jones NL. To tell or not to tell: primary care patients' disclosure deliberations. Arch Intern Med 2005; 165: 2378-83.
29 Sankar P, Mora S, Merz JF, Jones NL. Patient perspectives of medical confidentiality: a review of the literature. J Gen Intern Med 2003; 18 659-69.

30 Angermeyer MC, Daumer $\mathrm{R}$, Matschinger $\mathrm{H}$. Benefits and risks of psychotropic medication in the eyes of the general public: results of a survey in the Federal Republic of Germany. Pharmacopsychiatry 1993; 26: 114-20.

31 Double D. The limits of psychiatry. BMJ 2002; 324: 900-4.

32 Szasz TS. The myth of mental illness. Am Psychol 1960; 15: 113-8.

33 Sarkar SP. A British psychiatrist objects to the dangerous and severe personality disorder proposals. J Am Acad Psychiatry Law 2002; 30: 6-9.

34 American Psychiatric Association. The Principles of Medical Ethics with Annotations Especially Applicable to Psychiatry. APA, 2001.

35 Post JM. Ethical considerations in psychiatric profiling of political figures. Psychiatr Clin North Am 2002; 25; 635-46.

\title{
Medical psychotherapy: a specialty for now
}

\author{
Chris Mace, ${ }^{1}$ Kevin Healy ${ }^{2}$
}

The Psychiatrist (2011), 35, 301-304, doi: 10.1192/pb.bp.110.032599

\begin{abstract}
University of Warwick; ${ }^{2}$ West London Mental Health NHS Trust

Correspondence to Kevin Healy (kevin.healy@wlmht.nhs.uk)

First submission 13 Sep 2010, final revision 15 Dec 2010, accepted 28 Jan 2011

Summary If many patients having multiple difficulties are to receive the integral and efficient psychotherapies they require, they need attention from psychiatrists who have specialised training in psychotherapy. This paper sets out the roles to which existing holders of the Certificate of Completion of Training have already moved to, as they work with patients, families, teams and organisations. The General Medical Council has recognised that the understanding of medical psychotherapists is also vital to the future clinical teaching of all psychiatric trainees. This paper summarises key components of the knowledge and roles of future specialists in medical psychotherapy. It recommends that the term 'medical psychotherapy' be used widely to clearly differentiate psychiatrists with this training and these responsibilities from non-medical psychotherapists.
\end{abstract}

Declaration of interest None.
Six psychiatric specialties are listed in the legislation that gave overall responsibility for psychiatric training to the Postgraduate Medical Education and Training Board. ${ }^{1}$ They will be familiar as the options for specialty training from year 4 (ST4): general psychiatry, child and adolescent psychiatry, forensic psychiatry, old age psychiatry, psychiatry of learning disability, and psychotherapy. Doubts were raised about the adequacy of this list as a map of 21st-century psychiatry when the issue of the number of Certificates of Completion of Training (CCTs) in psychiatry was considered during an abortive attempt at reform. ${ }^{2}$ Although the number of CCTs is now unlikely to change, the current wider range of faculties and sections within the Royal College of Psychiatrists provides a more accurate map of specialism within psychiatry.

\section{From psychotherapy to medical psychotherapy specialty}

Among the CCT-bearing specialties, one is unique. 'Psychotherapy' is not descriptive of the types of patients seen, as is the case with the other five psychiatric specialties, but is instead descriptive of the therapeutic activity undertaken. Psychotherapy is not limited to clinical work that is only undertaken by psychiatrists. Indeed, as the importance of psychological therapies in the care of people with mental health problems grows, this is increasingly the case. As the range of professionals providing psychotherapy expands, so does the scope for confusion concerning the different expertise of the professionals delivering it. After due internal discussion and endorsement, the College has applied to the Department of Health for the psychiatric 